\title{
SEGURIDAD E HIGIENE INDUSTRIAL EN LAS PYMES DE HERMOSILLO, SONORA, MÉXICO.
}

\author{
Safety and industrial hygiene in PYMES \\ From Hermosillo, Sonora, Mexico.
}

EPISTEMUS

ISSN: 2007-8196 (electrónico)

ISSN: 2007-4530 (impresa)

Alberto Olguín Moreno ${ }^{1}$

Sergio Ramón Rossetti López ${ }^{2}$

J. Fernando García Arvirzu ${ }^{3}$

Manuel Arturo Coronado García 4

Recibido: 12 de septiembre de 2019,

Aceptado: 30 de noviembre del 2019

Autor de Correspondencia:

Alberto Olguín Moreno

Correo:

\section{Resumen}

En el presente artículo se realiza un estudio descriptivo de la situación actual de las pequeñas y medianas empresa del sector industrial que operan en la ciudad de Hermosillo, Sonora, México, con el objetivo de conocer el grado de aplicación de la normatividad existente en materia de seguridad e higiene. Para ello, tomando como referencia las normas oficiales mexicanas STPS-001, STPS-002, STPS-017 y STPS-021, se diseñó y aplicó un instrumento de cuestionario para obtener información sobre las condiciones de trabajo en materia de seguridad e higiene. La forma en que se llevó a cabo la selección de las empresas fue a través de la página de Internet de la Secretaría de Economía de México, tomando como población objetivo el listado pequeñas y medianas empresas del sector industrial, publicado en el sitio web. Posteriormente, a través de un muestreo estratificado se seleccionaron las pequeñas y medianas empresas. Para realizar el procesamiento y análisis estadístico de los datos, se utilizó el software Minitab 17. Con lo anterior, se obtuvieron algunos resultados sobresalientes de la investigación, por ejemplo, conocer qué porcentaje de pequeñas y medianas empresas industriales en la ciudad de Hermosillo aplican la normatividad de limpieza y ventilación. Al finalizar, se presentan algunas conclusiones y recomendaciones.

Palabras clave: seguridad, higiene, pequeñas y medianas empresas, normativas, industrial. clasificación JEL: L0, M0, K22

\section{Abstract}

This article presents a descriptive study of the current situation of small and medium industrial company that operate in the city of Hermosillo, Sonora, Mexico, with the aim of knowing the degree of application of the existing regulations in occupational safety and hygiene. To do this, taking as a reference the mexican official standards STPS-001, 002-STPS, STPS-017 and STPS-021, it was designed and applied a questionnaire tool to obtain information on conditions of work in the field of health and safety. The way in which the selection of companies was carried out was through the Internet of the office of economy in Mexico, taking as a target population the list of small and médium sized enterprises in the industrial sector, published on the web site. Subsequently, small and médium sized enterprises were selected through stratified sampling.To make this process and statistical analysis of the data, the software Minitab 17 was used. With this, some outstanding research results were obtained, for example, know what percentage of small and médium sized industrial enterprises in the city of Hermosillo apply the standards of cleanliness and ventilation. At the end, some conclusions and recommendations are presented.

Keywords: safety, hygiene, small and medium-sized companies, regulations, industrial. JEL classification: LO, MO, K22 


\section{INTRODUCCIÓN}

Las pequeñas y medianas empresas (PYMES), son organizaciones que generalmente se constituyen con poca infraestructura física, humana y con el mínimo capital de trabajo. Tal es el caso, que en la mayoría de éstas se enfocan en sobrevivir en los primeros años posteriores a su creación, para luego buscar consolidarse. Al igual que en todas las demás organizaciones, en las PYMES existen condiciones que deben prevalecer en los centros de trabajo, las cuales están reguladas bajo disposiciones legales, de tal suerte que al cumplir con los lineamientos que marca la ley, tanto el trabajador como el patrón se benefician con las bondades que se obtienen mediante la implantación de las normas que propone la higiene y seguridad industrial.

De esta forma, en el presente trabajo se realiza un diagnóstico de la situación actual en materia de seguridad e higiene de las pequeñas y medianas empresas industriales, establecidas en la ciudad de Hermosillo, Sonora, México. Lo anterior, con el propósito de conocer la importancia que tiene éste rubro para dichas empresas y en qué grado están aplicando o no la normativa regulatoria existente, para lo cual, se harán una serie de recomendaciones pertinentes con la finalidad de mejorar su funcionamiento, en aras de disminuir los problemas generados por actos $y$ condiciones inseguras, tales como accidentes o incapacidades de trabajo, a las que están expuestos los trabajadores y que en algunos casos son provocadas por el mismo personal debido a la falta de conocimientos en lo que a este tema se refiere.

Los accidentes de trabajo pueden ocurrir en cualquier momento, es por ello, que deben de eliminarse las condiciones y actos inseguros para así poder evitarlos. Según información obtenida de la Secretaría del Trabajo y Previsión Social en México (2017), en el año 2016 en México se tuvieron 394,202 accidentes de trabajo, 12,622 enfermedades de trabajo y 28,425 incapacidades de trabajo. Para el año 2017, estas cifras se incrementaron a

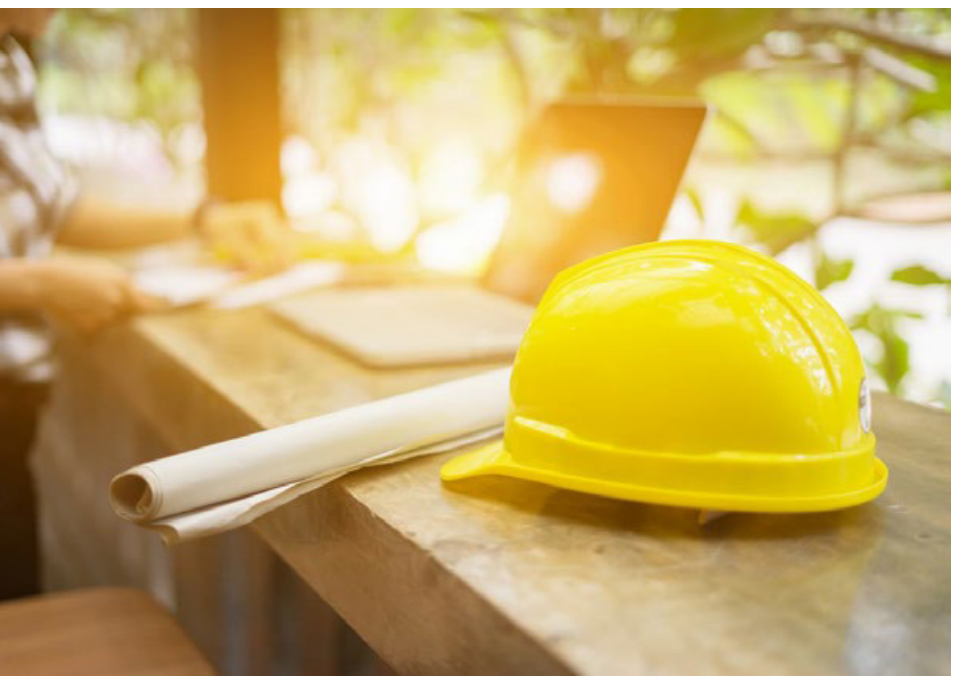

410,266 accidentes de trabajo, 14,159 enfermedades de trabajo y 29,856 incapacidades de trabajo.

En el contexto del Estado de Sonora, en el año 2016 se tuvieron 13,961 accidentes de trabajo, 579 enfermedades de trabajo y 1249 incapacidades. Para el año 2017 estas cifras se incrementaron 14,848 accidentes de trabajo, 679 enfermedades de trabajo y 1382 incapacidades. En este sentido, las fábricas pequeñas son un problema debido a que se estima que ocurren cerca de 2 millones de lesiones incapacitantes de trabajo, al año, el $30 \%$ aproximadamente, es decir 600,000 se produjeron en fábricas que tenían programas de seguridad industrial; mientras que el $70 \%$, es decir, $1,400,000$ se produjeron en instalaciones que no tenían programa alguno de seguridad industrial (Organización Internacional del Trabajo, 2019).

Otra referencia importante es que el número de trabajadores muertos o lesionados en su trabajo comenzó a alborear en la conciencia pública a mediados del siglo XIX, esto se empezó a dar en número creciente pues de los pequeños talleres empezaron a trabajar en las fábricas más grandes, contando con maquinaria nueva trayendo consigo nuevos peligros y nuevas lesiones. A pesar de la creciente preocupación por la seguridad, había falta de unidad entre la industria, los trabajadores y el gobierno. Al comienzo, los patronos asumieron solamente un grado limitado de responsabilidad y obligaciones por los accidentes; en su defensa contra demandas judiciales por daños y perjuicios alegaban negligencia o descuido por parte del trabajador o su ayudante, o que el trabajador lesionado había asumido el riesgo. Pero la creciente ola de accidentes llevó a una legislación más estricta para proteger al trabajador, constituida por las hoy bien conocidas leyes de indemnización de los trabajadores.

Para una mayor efectividad, el objetivo de los esfuerzos hacia la seguridad debía centrarse en el control de los riesgos más bien que en los "accidentes". Contemplados bajo esta luz, resulta claro que los objetivos de la seguridad deben orientarse a la identificación de los riesgos, la determinación de su significado, la evaluación de las medidas correctivas disponibles y la selección de los remedios óptimos. Cuando se sigue este camino, al final se encuentra la reducción de los acontecimientos no deseados hasta llegar a un mínimo inevitable. Si lo analizamos desde el punto de vista de la nación o de un patrón individual, se pueden lograr ahorros significativos en sufrimiento de seres humanos y en utilidades a través de esfuerzos efectivos encaminados a fomentar la seguridad (Grimaldi y Simonds, 2001).

Toda industria contrae obligaciones al momento de establecerse, por lo cual es importante que se preocupe por el bienestar tanto del trabajador como de las instalaciones físicas, de tal forma que procure eliminar riesgos de trabajo que aseguren la integridad de los individuos; por ello en este trabajo, se investigan las condiciones bajo las cuales se labora en las PYMES asociadas a la Cámara Nacional de la Industria de la Transformación (CANACINTRA) de la ciudad 
de Hermosillo, Sonora, para así constatar si se siguen los lineamientos señalados por la higiene y seguridad industrial en lo referente a las condiciones físicas de pisos y paredes y señalización indispensable del centro de trabajo, capacitación a los trabajadores en primeros auxilios y en la prevención y protección de incendios, de tal forma que todo esto ayude a la eliminación de riesgos.

A manera de diagnóstico y con datos proporcionados por la unidad médica de salud en el trabajo del Instituto Mexicano del Seguro Social (IMSS) zona Hermosillo, los accidentes de trabajo consumados en el año 2017 (ver tabla 1) toman el lugar número uno de entre los riesgos laborales lo cual nos indica que es necesario seguir lineamientos que nos ayuden a disminuir o eliminar dichos riesgos que garanticen el bienestar del trabajador.

Tabla 1. Riesgos de trabajo y sus consecuencias en el año 2017, para el estado de sonora.

$$
\text { CONCEPTO }
$$

\section{NÚMERO}

Riesgos de trabajo:

\begin{tabular}{|l|l|}
\hline Accidentes de trabajo & 14,848 \\
\hline Accidentes de trayecto & 3,979 \\
\hline Enfermedades de trabajo & 679 \\
\hline Días de incapacidad otorgados & 498,931 \\
\hline Defunciones & 28 \\
\hline
\end{tabular}

Fuente: Elaboración propia basado en la Secretaría del Trabajo y Previsión Social, 2017.

\section{MARCO TEÓRICO Y NORMATIVO}

La Higiene y Seguridad Industrial es una técnica que tiene como finalidad evitar que ocurran accidentes y enfermedades de trabajo mediante la detección, evaluación y control de los riesgos laborales. El término enfermedad profesional o de trabajo implica que el cuadro

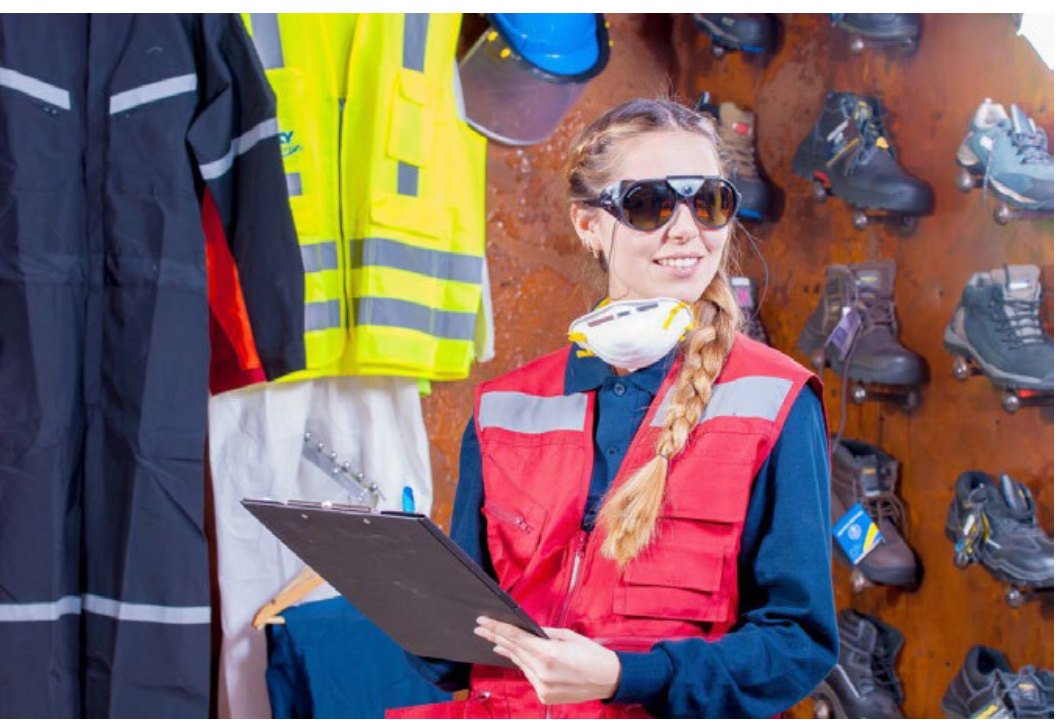

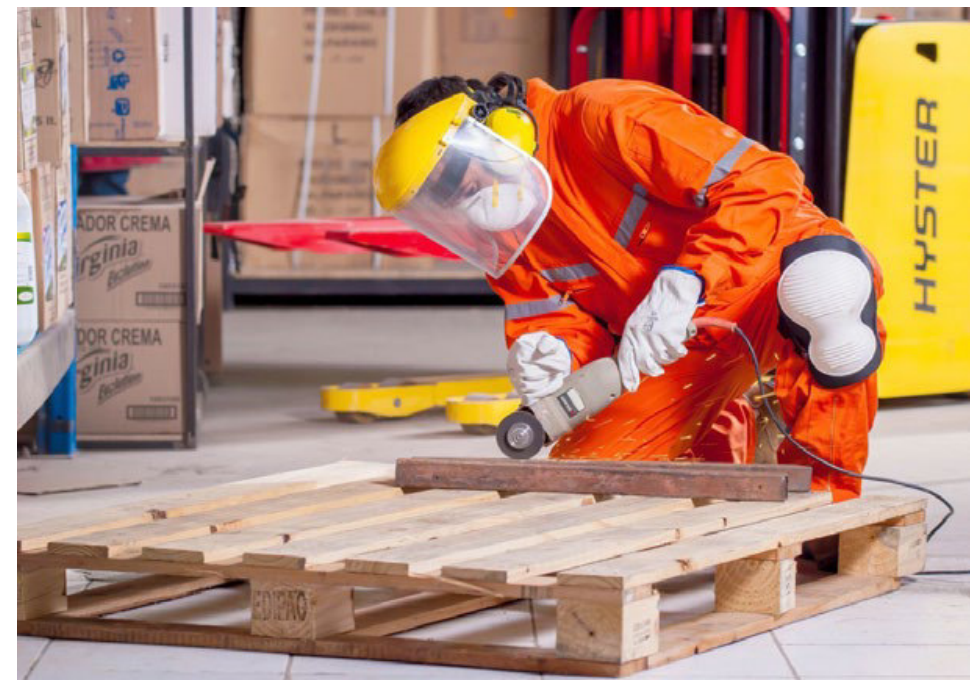

patológico se deriva del trabajo $u$ oficio del individuo. La intoxicación ocupacional es uno de los principales factores que afectan la salud de los trabajadores; para cada enfermedad profesional hay un agente etiológico determinado. El periodo de incubación, o sea el tiempo transcurrido entre la contaminación y la aparición de los primeros síntomas, puede variar mucho más para las enfermedades profesionales que para otras enfermedades. En ocasiones para contraer una enfermedad profesional es necesario el contacto con una sustancia durante meses o años. El resfriado común y la gripe son las menos graves del grupo de enfermedades respiratorias pero causan la mayor pérdida de tiempo, es decir, ausentismo en el trabajo (Jelambi, 1967).

Es importante considerar que existen riesgos laborales en cada centro de trabajo, los cuales hay que conocer así como la forma de combatirlos, ejemplo de esto es tener en cuenta que, para que un incendio estalle en un lugar es necesario que concurran en él tres elementos: que haya oxígeno, algún material combustible y cierta cantidad de calor (para que el fuego prenda es necesaria cierta temperatura mínima, pero una vez que ha prendido produce por sí mismo la temperatura necesaria para mantenerse). Si se elimina cualquiera de los tres elementos, el incendio no puede declararse, o si ya se declaró, no puede continuar. De ahí que los métodos de prevención de incendios consistan en lo esencial en la reducción o la eliminación de uno de estos tres elementos. No obstante, dos de ellos, el oxígeno y algún material combustible están presentes en prácticamente todos los establecimientos industriales, y por ello es imprescindible asegurar de una u otra manera que el tercer elemento "el calor" no alcance jamás el nivel suficiente para iniciar un incendio (Organización Internacional del Trabajo, 2019).

Por otro lado, es de suma importancia tener presente que los polvos de materia orgánica como harina, azúcar, almidón y corcho, y algunos polvos metálicos, como los de aluminio y magnesio, pueden resultar explosivos al mezclarse con el aire (Oficina Internacional del Trabajo, 
2019). Cabe mencionar que el escenario ideal es que las instalaciones del centro de trabajo hayan sido construidas con resistencia al fuego procurando que la estructura del edificio no prenda fácilmente y de esta forma no sea posible su fácil propagación, pero en realidad en muchos de los casos el edificio no ha sido construido especialmente para sus necesidades de trabajo sino más bien ha tenido que ser adecuado.

La Organización Internacional del Trabajo (OIT) menciona unas reglas que han de considerarse al momento de construir el edificio, y se mencionan a continuación:

1.Ninguna parte del edificio deberá estar alejada de una salida al exterior y su distancia de ella deberá ser en función del grado de riesgo existente.

2. Cada piso deberá tener al menos 2 salidas, suficientemente grandes, protegidas contra las llamas y el humo y bien separadas entre sí.

3. Las escaleras de madera, las escaleras de caracol, los ascensores y las escaleras de mano no deberán ser considerados como salidas de emergencia.

4.Las salidas deberán mantenerse siempre sin obstrucciones.

5. El acceso a las salidas deberá mantenerse siempre sin obstrucciones. Las escaleras exteriores y de escape para caso de incendio no deberán dar a patios internos o pasajes sin salida.

Es esencial efectuar inspecciones periódicas en las empresas para descubrir los riesgos de incendio y cerciorarse de que todo el equipo de lucha contra incendios se encuentra en buen estado. Asimismo, es conveniente organizar periódicamente simulacros para asegurarse de que todos los trabajadores sepan cómo utilizar el equipo extintor, dónde se encuentra la salida más cercana y cómo abandonar el edificio en orden. Durante los simulacros debe comprobarse si el número de salidas es suficiente para que el establecimiento pueda rápidamente ser evacuado por todo el personal. Sin embargo, no debe olvidarse que los simulacros de incendio son caros, pues interrumpen la producción repentinamente y puede necesitarse cierto tiempo para recobrar el ritmo. Por consiguiente, este método debe utilizarse en forma juiciosa.

Para el control de riesgos laborales se cuenta con una serie de lineamientos, los cuales deben llevarse a cabo con el fin de reducir los problemas ocasionados por actos y condiciones inseguras. Es fácil saber cuáles son los puntos que se deben cumplir ya que todo está regido por reglamentos y normas. Uno de ellos es el Reglamento Federal de Seguridad y Salud en el Trabajo (RFSST), el cual rige en todo el territorio nacional y tiene por objeto proveer en la esfera administrativa a la observancia de la Ley Federal del Trabajo (LFT) en materia de seguridad e higiene y lograr de este modo disminuir los accidentes y enfermedades que se producen $u$ originan en los centros

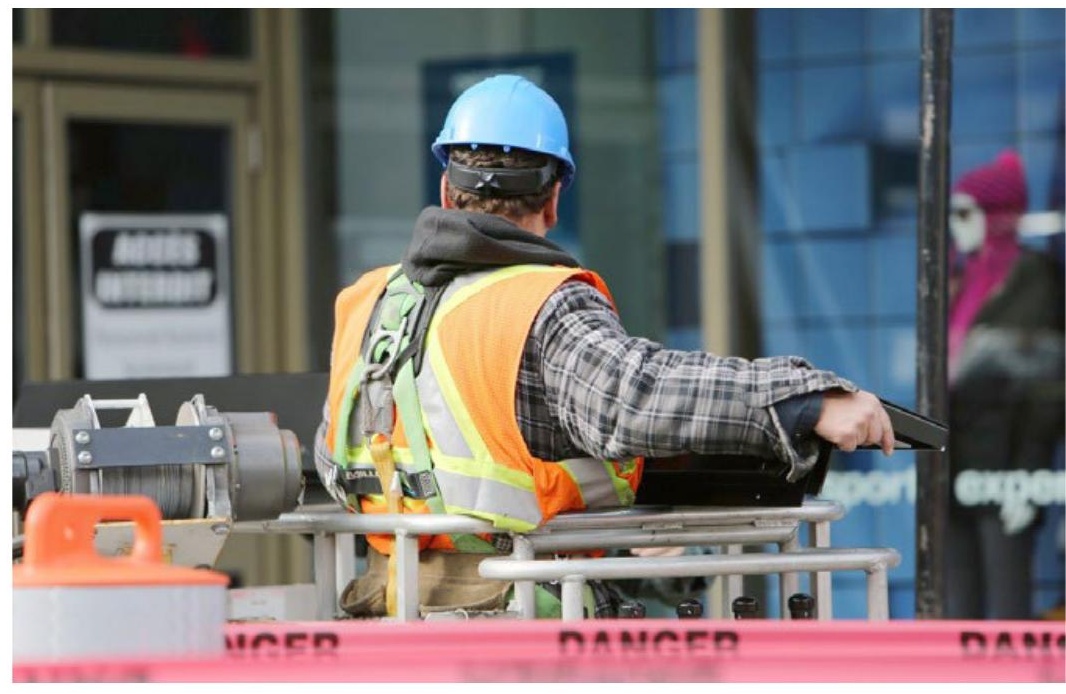

de trabajo.

Además existen Normas Oficiales Mexicanas (NOM) 1 de la Secretaría del Trabajo y Previsión Social que rigen las condiciones de seguridad e higiene para cada centro de trabajo y por lo tanto deben de ponerse en práctica. Están hechas para beneficio tanto del patrón como de los trabajadores y de acuerdo con la actividad de cada empresa serán las normas aplicables a cada uno de ellos.

Debido a los objetivos de esta investigación las normas oficiales mexicanas que se considerarán son las emitidas por la secretaría del trabajo y previsión social, y se mencionan a continuación:

- NOM-001-STPS-2008. Edificios, locales, instalaciones y áreas en los centros de trabajo - Condiciones de seguridad e higiene.

- NOM-002-STPS-2010. Condiciones de seguridad Prevención, protección y combate de incendios en los centros de trabajo.

- NOM-017-STPS-2008. Equipo de protección personal Selección, uso y manejo en los centros de trabajo.

- NOM-021-STPS-1993. Relativa a los requerimientos y características de los informes de los riesgos de trabajo que ocurran, para integrar las estadísticas.

\section{HIPÓTESIS}

H1: Las PYMES industriales de la ciudad de Hermosillo, Sonora, México inscritas a la CANACINTRA, aplican las normatividades que rigen a los centros de trabajo en materia de higiene y seguridad industrial.

$\mathrm{H} 2$ : Las pequeñas empresas del sector industrial de la ciudad de Hermosillo, Sonora, México inscritas a la CANACINTRA, aplican en igual número las normatividades que rigen los centros de trabajo en materia de higiene y seguridad industrial, en comparación con las medianas empresas del mismo sector en Hermosillo, Sonora.

1 Normas Oficiales Mexicanas (2018). Disponible en: http://www.stps.gob. $\mathrm{mx} / \mathrm{bp} /$ secciones/conoce/marco_juridico/noms.html 


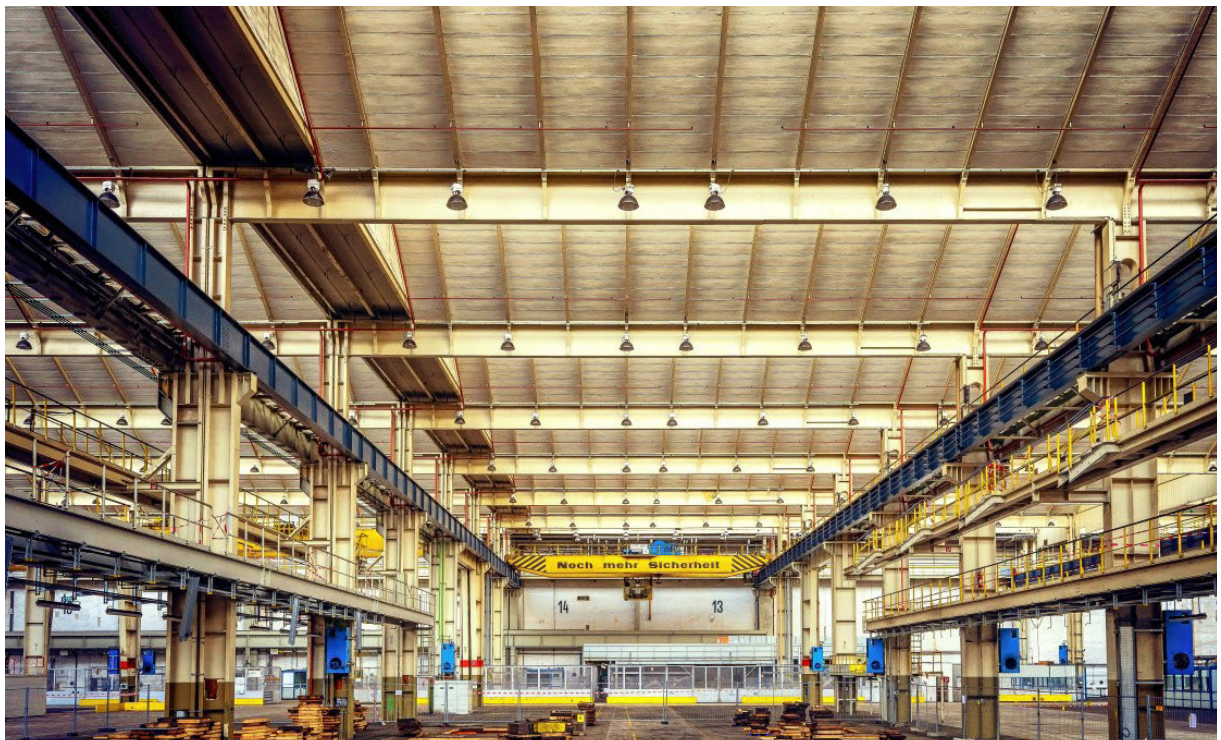

METODOLOGÍA

La presente investigación se lleva cabo mediante un estudio de tipo descriptivo con un enfoque cuantitativo. Descriptivo debido a que se estudia una situación en particular en condiciones naturales para deducir un ambiente o circunstancias que se están presentando y cuantitativo ya que se lleva a cabo recolección de datos, análisis estadístico tabular, gráfico y pruebas de hipótesis para su posterior interpretación en los resultados.

Para la realización de esta investigación se consideraron las pequeñas y medianas empresas industriales asociadas a la Cámara Nacional de la Industria de la Transformación (CANACINTRA) de la ciudad de Hermosillo, Sonora. La investigación se enfocará a temas de relación directa con higiene y seguridad industrial siendo ésta un área de estudio muy importante para las PYMES debido a que por su tamaño son empresas que generalmente los dirigentes de dichos establecimientos no muestran interés por seguir con los lineamientos que ésta marca. Además, son empresas que requieren de apoyos y estudios que les muestren las ventajas de seguir muy de cerca lo relacionado con este tema y así ser cada día más competitivos; y que mejor forma que iniciando por cumplir con lineamientos legales como reglamentos y normas oficiales mexicanas que les permitan ofrecer un producto de calidad, elaborado tomando en cuenta las medidas de seguridad necesarias tanto para el recurso humano como el material.

Como ya se mencionó, se delimitó la población de estudio a las empresas asociadas a la CANACINTRA y que considerando el tamaño de éstas se estratificó dicha población en pequeñas y medianas empresas industriales debido a que se consideró que este sector es el que está medianamente involucrado con temas relacionados con higiene y seguridad industrial, y de esta manera hacer posible un comparativo más acorde a las características similares que tienen entre sí las PYMES, pues hacer comparaciones entre empresas micro o grandes sería hacerlo inequitativamente. Además, es posible considerar que las empresas que forman parte de los asociados a la cámara muestran cierto interés de progreso, pues al estar inscritos a ella gozan de beneficios como asesoría legal, capacitación y diversos servicios que ayudan a las empresas industriales a estar actualizadas y ser competitivas en su campo de acción.

El directorio de socios se obtuvo del sitio en Internet del sistema empresarial mexicano, en donde se lista la totalidad de las empresas asociadas, por lo que se procedió a clasificarlas de acuerdo con el tamaño de la empresa. El tamaño de empresa se consideró en base al número de empleados, en cual no es proporcionado en la totalidad de las empresas incluidas en el listado por lo que éstas no considerarán para su clasificación y por lo tanto no podrán ser objeto de estudio.

La clasificación de las PYMES se realizó utilizando los rangos manejados por la Secretaría de Economía y fueron consultados en su sitio de Internet, 2 la cual se muestra a continuación:

- De 11 a 50 empleados se considera como pequeña empresa.

- De 51 a 250 empleados es considerada mediana empresa.

Las empresas que tienen como máximo 10 empleados son consideradas microempresas y las que exceden los 251 empleados entran en el rango de grandes empresas. Tomando como referencia los rangos anteriormente señalados, se procedió a realizar la clasificación del listado de empresas asociadas a la cámara. A continuación, se muestran los datos en la tabla 2:

Tabla 2. Clasificación de las empresas asociadas a canacintra según tamaño.

\begin{tabular}{l|l}
$\begin{array}{l}\text { TIPO DE EMPRESA } \\
\text { INDUSTRIALES }\end{array}$ & $\begin{array}{c}\text { CANTIDAD DE } \\
\text { EMPRESAS }\end{array}$ \\
\hline Pequeñas & 13 \\
\hline Medianas & $\underline{18}$ \\
\hline Total & $\mathbf{3 1}$
\end{tabular}

Fuente: Elaboración propia.

Una vez realizada la estratificación anterior, se procedió a considerar como población de estudio sólo a las pequeñas y medianas empresas, partiendo de ellas se hizo un reacomodo para considerar sólo a las empresas

2 Secretaría de Economía (2019). Disponible en: http://www.economia. gob.mx 
que por su actividad productiva se consideran como industriales. Obteniéndose finalmente como población de estudio a 13 pequeñas empresas industriales y 18 medianas industrias, dando esto una población total de 31 elementos. Conociendo estos datos se procedió al cálculo del tamaño de muestra, mediante la siguiente fórmula:

$$
\begin{gathered}
\mathrm{n}=\frac{\sum_{i=1}^{L} N_{i} p_{i} q_{i} / w_{i}}{\mathrm{ND}+\frac{1}{N} \sum_{i=1}^{L} N_{i} p q_{i}} \\
w_{i}=N i / N^{\mathrm{D}}=\frac{B^{2}}{Z_{1-\alpha 2^{2}}}
\end{gathered}
$$

Donde:

$w_{i}=$ fracción de observaciones asignadas al estrato $i$

$p_{i}=$ proporción poblacional del estrato $i$

$B=$ error de estimación

Utilizando un margen de error de $5 \%$ y un $95 \%$ de confiabilidad en dicha fórmula.

Se determinó un $95 \%$ de confiabilidad debido a que con dicho porcentaje se asegura que la muestra estudiada represente en lo posible a las características de la población en general. Estadísticamente es óptimo y recomendable utilizar dicho porcentaje. Un porcentaje menor, no daría la confianza que el estudio requiere para considerar la información que se obtenga para estudios posteriores o tomar medidas de acción pertinentes. Y si se pretendiera utilizar una cantidad mayor prácticamente se tendría que aplicar la encuesta al total de la población de estudio.

El 5\% de error a considerar es también estadísticamente reconocido como un margen aceptable de error, pues si se considera una cantidad mayor no sería muy confiable la información obtenida mediante la investigación. De igual manera un porcentaje menor haría que el tamaño de muestra aumentara y nos llevaría con ello a muestrear casi el $100 \%$ de la población. Empleando la fórmula se obtuvo los siguientes tamaños de muestra:

- Pequeñas empresas industriales: 6

- Medianas empresas industriales: 8

Una vez determinado el tamaño de la muestra, se seleccionaron las empresas a encuestar por medio de una tabla de números aleatorios, utilizando un método de selección de muestreo aleatorio simple, el cual indica que cada elemento de la población tiene la misma probabilidad de ser seleccionado dentro del grupo al que corresponde, siendo este sin reemplazo, es decir que se irán sacando aleatoriamente cada empresa hasta hacer el listado que incluya la totalidad de las empresas que conformarán la muestra y a quienes se les aplicará la encuesta para obtener la información necesaria (Cochran, 1980).

\section{INSTRUMENTO DE MEDICIÓN}

Se elaboró una carta de presentación que se antepuso al formato de la encuesta, la cual pretende propiciar la disponibilidad por parte del encuestado a responderla, así como solicitar que la información que se proporcione sea

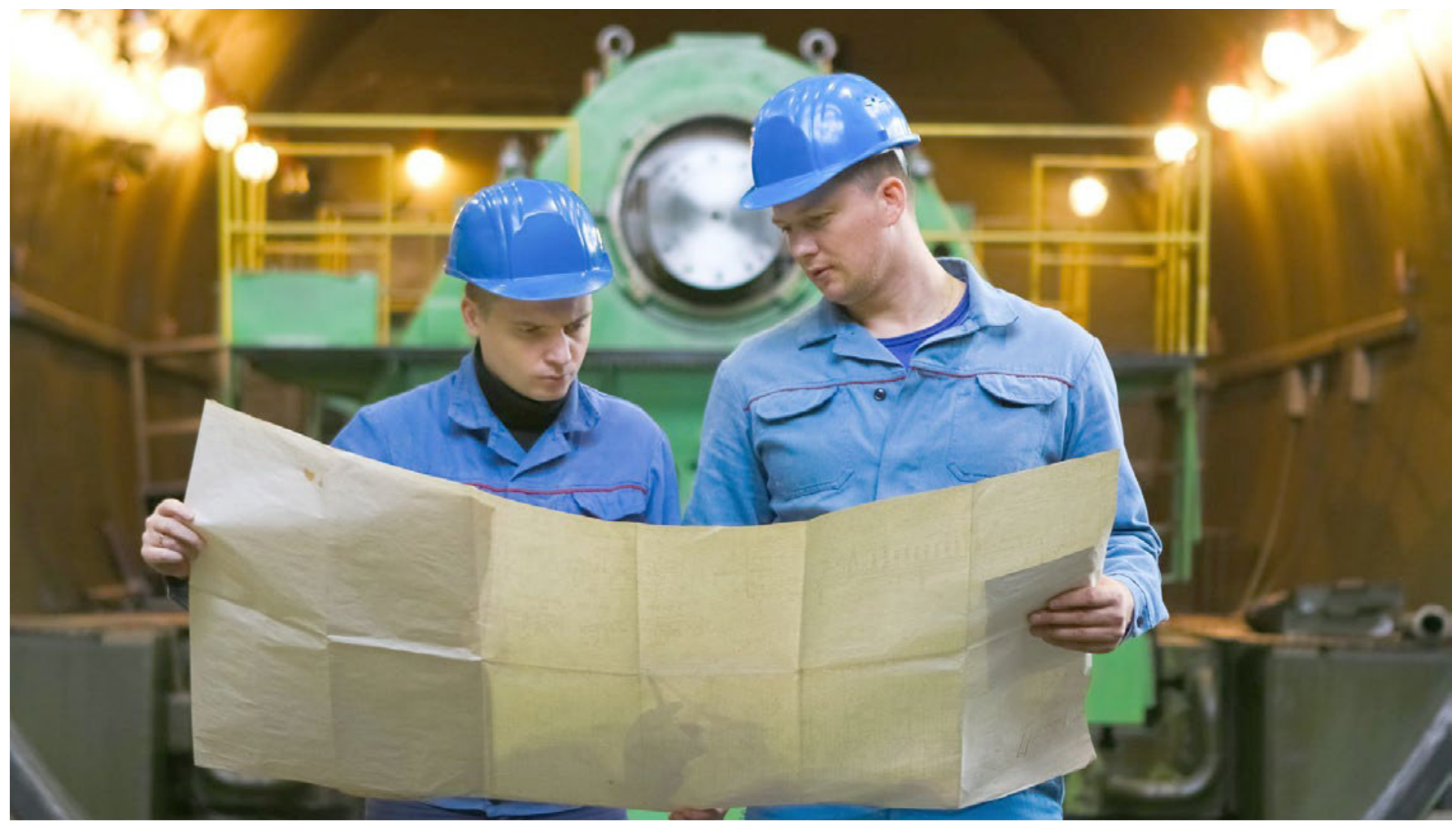


fidedigna para que el análisis esté en lo posible apegado a la realidad y a la situación actual por la cual atraviesan las PYMES. El formato de la encuesta se elaboró para la obtención de la información, y se muestra en el anexo, el cual se realizó tomando en cuenta los aspectos que se mencionan en las normas oficiales mexicanas mencionadas en el marco normativo de este documento y al reglamento federal de seguridad y salud en el trabajo, publicado en el Diario Oficial de la Federación el 13 de noviembre de 2014.

En las preguntas, la clasificación de las respuestas será por atributos debido a que son preguntas con opción de respuesta cerrada. Como atributo se considerará los números 0 y 1 , en donde el 0 (cero) será considerado como fracaso (es decir, un no como respuesta); y el número 1 cuando se obtenga un Sí como respuesta.

\section{RESULTADOS}

Una vez concluida la etapa de recopilación de la información, se procedió a realizar el análisis de los datos, los cuales fueron manipulados en el paquete Microsoft Excel 2013 y el paquete Estadístico Minitab 17. En las siguientes tablas se presentan los resultados abreviados, esto con la finalidad de acotar la información y exponer aquellos más representativos, mismos que nos ayudarán a comprobar las hipótesis planteadas. En el anexo A2 se puede consultar la redacción de todas las preguntas.

Tabla 3. Porcentaje de pequeñas y medianas empresas que cumplen / no cumplen.

\begin{tabular}{|c|c|c|c|c|}
\hline & \multicolumn{2}{|c|}{ PEQUENAS EMPRESAS } & \multicolumn{2}{c|}{ MEDIANAS EMPRESAS } \\
\hline PREGUNTA & \% SÍ CUMPLE & \% NO CUMPLE & \% SÍ CUMPLE & \% NO CUMPLE \\
\hline 1 & 46 & 54 & 83 & 17 \\
\hline 5 & 92 & 8 & 100 & 0 \\
\hline 10 & 31 & 69 & 77 & 23 \\
\hline 14 & 56 & 44 & 100 & 0 \\
\hline 17 & 46 & 54 & 77 & 23 \\
\hline
\end{tabular}

Fuente: Elaboración propia

Como puede observarse en la tabla anterior, las empresas medianas dan un mayor cumplimiento a las normas y reglamentos que regulan el buen funcionamiento de las empresas industriales del país. Es importante hacer notar que el $83 \%$ de las medianas empresas y sólo el $46 \%$ de las pequeñas cuentan con registros de las verificaciones oculares realizadas al centro de trabajo, para identificar condiciones inseguras y reparar daños encontrados.

En lo referente a la pregunta 5 del cuestionario, nos permite identificar que el $100 \%$ de las medianas empresas se constata físicamente que los edificios no presentan deformaciones o daños, en tanto que las pequeñas empresas esta cifra disminuye al $92 \%$.

En lo relacionado con la pregunta 10, la cual se refiere a si se ha elaborado un cálculo para la determinación final del riesgo de incendio muestra que la medianas empresas dan un mayor cumplimiento que las pequeñas, debido a que se obtuvo una diferencia del $46 \%$, es decir la mayoría de las pequeñas empresas no determinan el costo de sus bienes en función de un siniestro, por lo tanto, no se está cumpliendo con la normatividad (la cual indica que deben de realizar un inventario de los activos susceptibles de consumirse por el fuego).

Para comparar los porcentajes obtenidos mediante la pregunta 14 , se muestra que un $56 \%$ de las pequeñas empresas y un $100 \%$ de las empresas medianas brinda capacitación en el uso de las herramientas de trabajo; lo cual muestra que más del $40 \%$ de las empresas muestreadas no aplican lo estipulado en la norma.

En la pregunta 17, el $77 \%$ de las medianas empresas manifestaron contar con 
registro de los avisos por riesgo de trabajo ocurridos en las áreas de trabajo, mientras que las pequeñas empresas sólo el $46 \%$ mantienen un registro de los hechos ocurridos en las instalaciones. De nueva cuenta, se puede advertir que las PYMES no cumplen con la reglamentación establecida por la STPS.

Con las estadísticas presentadas en la tabla 3 , puede inferirse que las medianas empresas industriales de la ciudad de Hermosillo cumplen en mayor porcentaje con lo que establecen las normatividades existentes en relación con las pequeñas empresas, lo cual no significa que las medianas cumplan correctamente o en lo posible con todos los lineamientos. La información recopilada permite identificar cuáles son los rubros que requieren ser atendidos con prioridad, pero también todos aquellos en los que se tiene que trabajar para tener centros de trabajo más seguros.

De esta forma, se pudo tener una idea de la situación actual en la que se encuentran las PYMES industriales de la entidad, la cual muestra que hay mucho por hacer para lograr cumplir sobre lineamientos legales y seguir las recomendaciones de la Higiene y Seguridad Industrial.

Con lo anterior se rechaza la hipótesis $\mathrm{H} 1$ del estudio, la cual afirma que las PYMES industriales de Hermosillo,
Sonora, México dan cumplimiento a las normatividades existentes en relación directa con Higiene y Seguridad Industrial.

En lo que respecta a la hipótesis $\mathrm{H} 2$ del estudio, se presentan estadísticos a través de pruebas de hipótesis para determinar la existencia de diferencias significativas en el cumplimiento de las normas en empresas medianas y pequeñas. Para ello, se agruparon las respuestas de los ítems por categorías, es decir, se agruparon en una primera categoría denominada "Recorrido por la empresa" las respuestas de las preguntas 1 , 2, 3, 4, 5, 6, 7 y 8, en una segunda denominada "Incendios" las respuestas de las preguntas 9, 10 y 11, en una tercer categoría "Equipo de protección" las respuestas de la preguntas $12,13,14$ y 15, y finalmente en la categoría "Estadísticas" las respuestas a las preguntas 16, 17, 18 y 19. Ver anexo A2.

En la siguiente tabla 4, se muestran las medias de respuestas a cada pregunta de instrumento, agrupadas por categoría. Por ejemplo en la primera columna "Recorridos por la empresa", en las pequeñas empresas se obtuvo un promedio de 4.77, es decir, en promedio las empresas pequeñas cumplen con 4.77 normas relacionadas a los recorridos por la empresa. El valor máximo posible es 8 (cumplen con todas las normas) y el mínimo 0 (no cumplen con alguna de las normas).

Tabla 4. Promedios de aplicación de normas en empresas pequeñas y medianas industriales por categoría.

\begin{tabular}{|c|c|c|c|c|}
\hline & RECORRIDOS POR LA EMPRESA & INCENDIOS & $\begin{array}{c}\text { EQUIPO DE } \\
\text { PROTECCIÓN }\end{array}$ & ESTADÍSTICAS \\
\hline Escala & $0-8$ & $0-3$ & $0-4$ & $0-4$ \\
\hline Pequeñas & 4.77 & 1.62 & 2.38 & 2.31 \\
\hline Medianas & 6.94 & 2.44 & 3.56 & 3.28 \\
\hline
\end{tabular}

Fuente: Elaboración propia

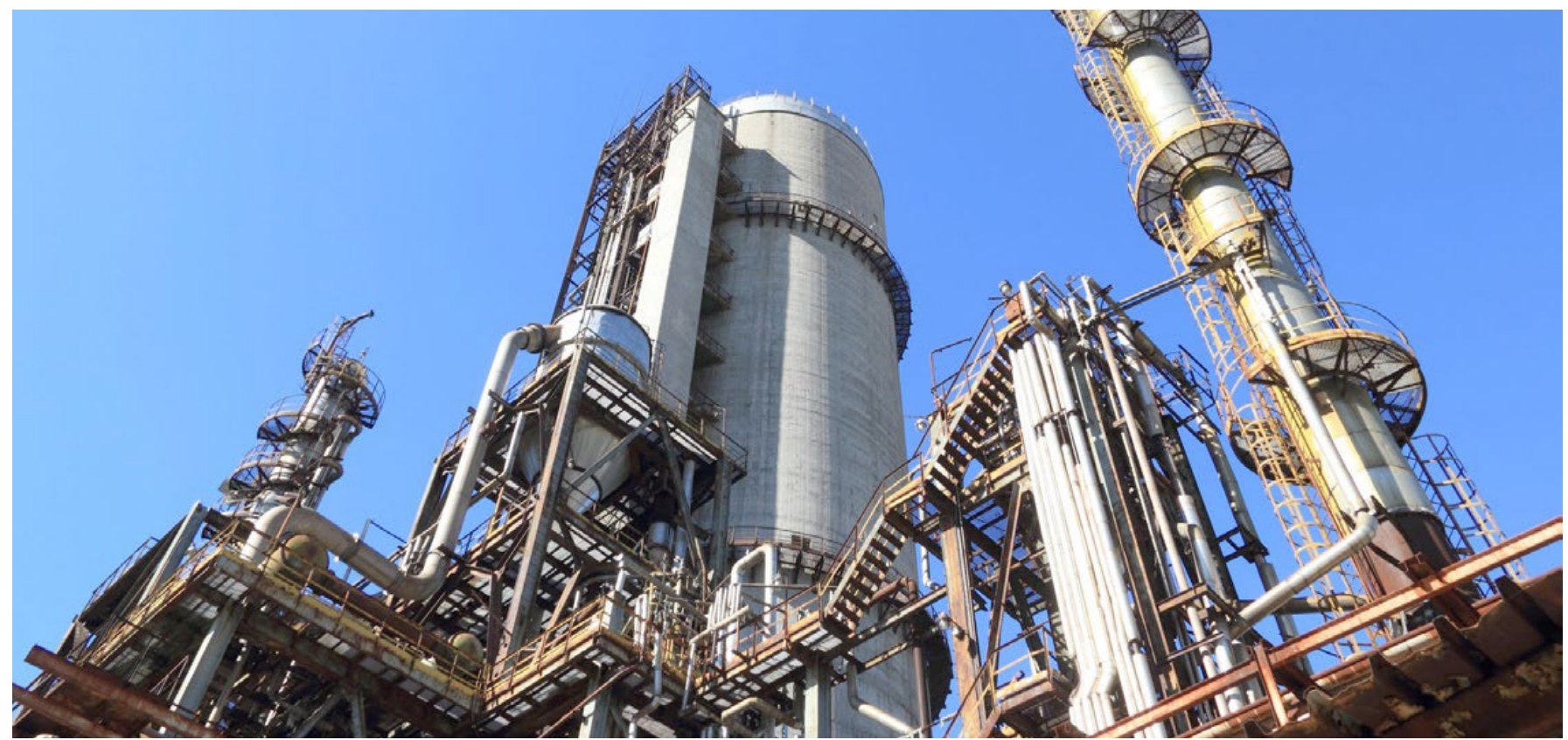




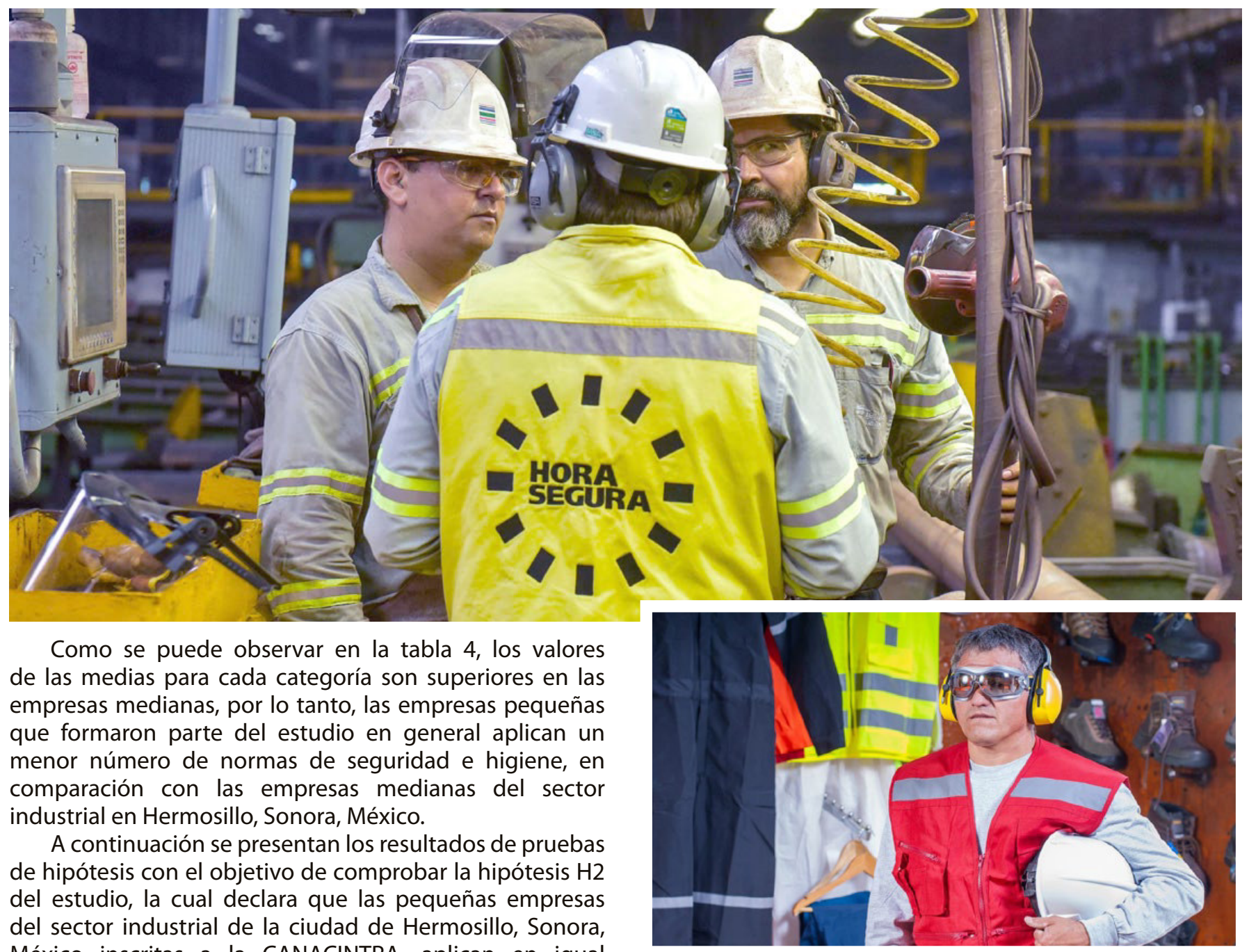

México inscritas a la CANACINTRA, aplican en igual número las normatividades que rigen los centros de trabajo en materia de higiene y seguridad industrial, en comparación con las medianas empresas del mismo sector en Hermosillo, Sonora.

Para ello, se utilizó la prueba estadística T para dos muestras independientes, la cual compara si las medias de dos grupos de datos independientes son diferentes.

Figura 1. Prueba T Recorridos por la empresa.

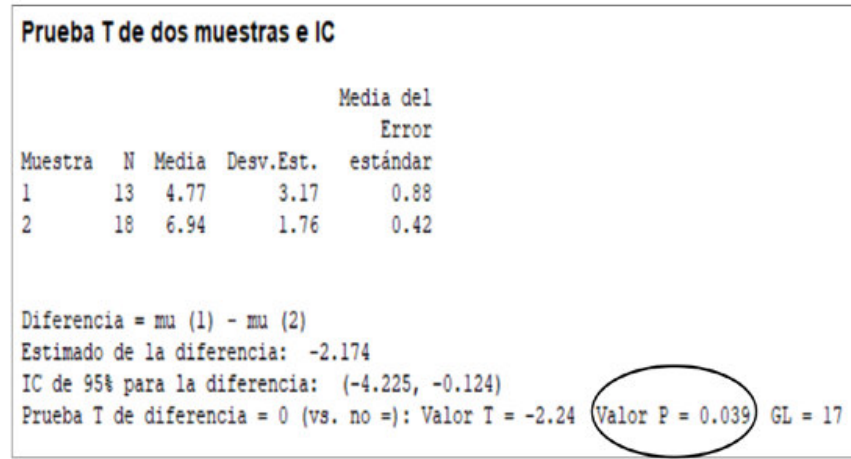

Fuente: Elaboración propia

Como se observa en la figura 1 , el valor $p=0.039$ es menor a un nivel de significancia $\alpha=.05$, por lo tanto existe evidencia para concluir que la diferencia entre las medias de las dos poblaciones no es igual a la diferencia hipotética $=0$, es decir, existe diferencia significativa entre la media del número de normas de "Recorridos por la empresa" entre las pequeñas y medianas empresas.

Figura 2. Prueba T Incendios.

Prueba T de dos muestras e IC

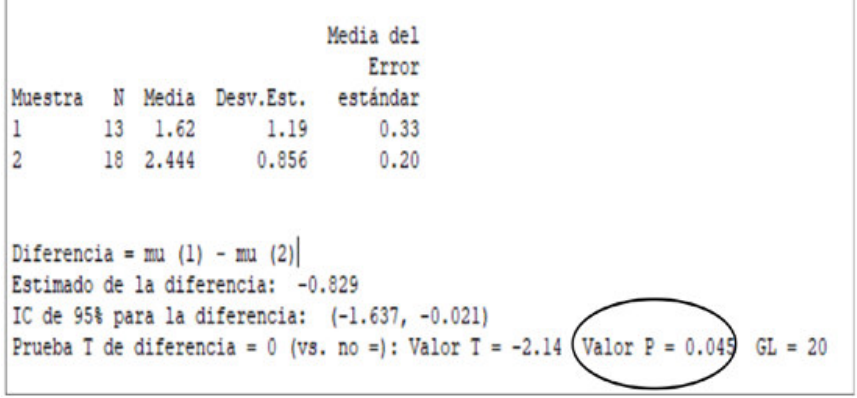

Fuente: Elaboración propia 
Como se observa en la figura 2 , el valor $p=0.045$ es menor a un nivel de significancia $\alpha=.05$, por lo tanto existe evidencia para concluir que la diferencia entre las medias de las dos poblaciones no es igual a la diferencia hipotética $=0$, es decir, existe diferencia significativa entre la media del número de normas de "Incendios" entre las pequeñas y medianas empresas.

Figura 3. Prueba T Equipo de protección.

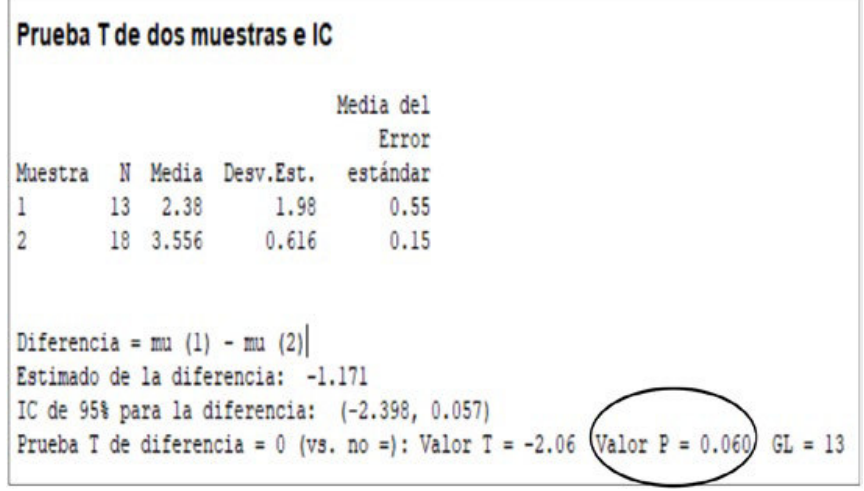

Fuente: Elaboración propia

Como se observa en la figura 3 , el valor $p=0.060$ es mayor a un nivel de significancia $\alpha=.05$, por lo tanto no existe suficiente evidencia para concluir que la diferencia entre las medias de las poblaciones es estadísticamente significativa, es decir, no se puede concluir que exista diferencia significativa entre la media del número de normas de "Equipo de protección" entre las pequeñas y medianas empresas.

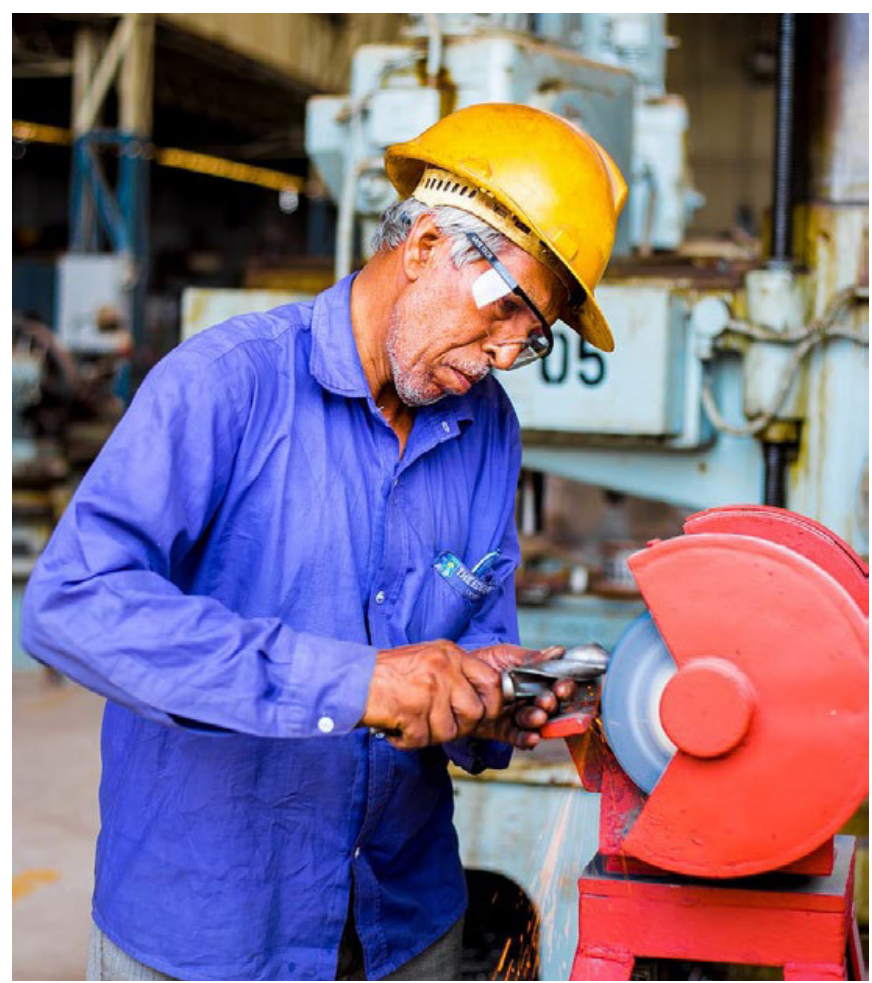

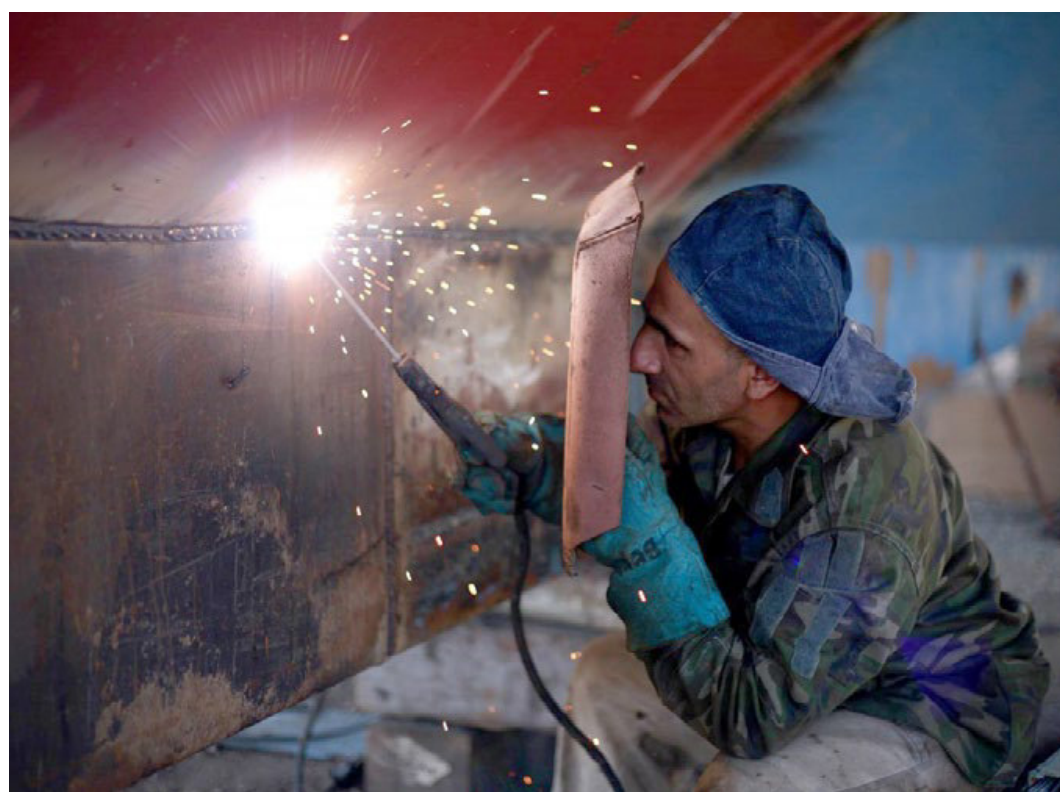

Figura 4. Prueba T Estadísticas.

Prueba T de dos muestras e IC

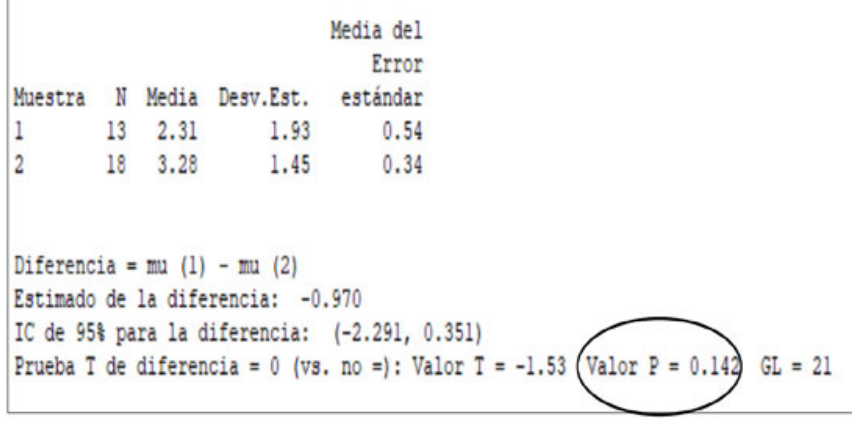

Fuente: Elaboración propia

Como se observa en la figura 4 , el valor $p=0.142$ es mayor a un nivel de significancia $\alpha=.05$, por lo tanto no existe suficiente evidencia para concluir que la diferencia entre las medias de las poblaciones es estadísticamente significativa, es decir, no se puede concluir que exista diferencia significativa entre la media del número de normas de "Estadísticas" entre las pequeñas y medianas empresas.

Con los resultados anteriores, no se acepta la hipótesis $\mathrm{H} 2$ del estudio, la cual indica que las pequeñas empresas del sector industrial de la ciudad de Hermosillo, Sonora, México inscritas a la CANACINTRA, aplican en igual número las normatividades que rigen los centros de trabajo en materia de higiene y seguridad industrial, en comparación con las medianas empresas del mismo sector en Hermosillo, Sonora. Por lo anterior, se concluye que existe una influencia en el número de normas y procedimientos en materia de seguridad e higiene, relacionada con el tamaño de las empresas, es decir, las empresas pequeñas en general cumplen con un número menor de normas en los rubros de "Equipos de protección" y "Estadísticas", en comparación con las empresas medianas. 


\section{CONCLUSIONES}

Retomando la hipótesis de trabajo y analizando los resultados obtenidos (proporciones de empresas que aplican los lineamientos relacionados con higiene y seguridad industrial) podemos decir que la hipótesis se acepta, es decir no se está dando cumplimiento a las normatividades existentes. De acuerdo con el análisis de los datos del capítulo anterior podemos inferir que las medianas empresas aplican en mayor proporción las normatividades sobre higiene y seguridad industrial en comparación con las pequeñas empresas en la ciudad de Hermosillo, Sonora, México.

Acerca de los registros que cada empresa debe de llevar y que exige cada una de las normas oficiales mexicanas, no se le da cumplimiento por parte de las medianas y tampoco por parte de las pequeñas empresas. Por otro lado, se deben de considerar los registros estadísticos en materia de seguridad como una base para obtener información de forma inmediata, que permita tomar decisiones sobre: rediseño de puestos de trabajo, reubicación de empleados, cursos de capacitación, distribución de planta, entre otros.

Consideramos que la CANACINTRA está comprometida a orientar a sus socios de tal forma que puedan implantar las medidas necesarias para estar preparados en caso de presentarse un incendio, pero de igual forma debe haber disponibilidad por parte de los responsables de dichas empresas para la implantación de estrategias que permitan el mejor uso de las instalaciones y el incremento de la seguridad y satisfacción de sus trabajadores; debido a que invertir en equipo o capacitación no es un gasto sino una inversión que otorgará seguridad a ambas partes.

Se recomienda revisar periódicamente que el equipo para la prevención y combate de incendios, así como la señalización básica del centro de trabajo esté ubicado en el

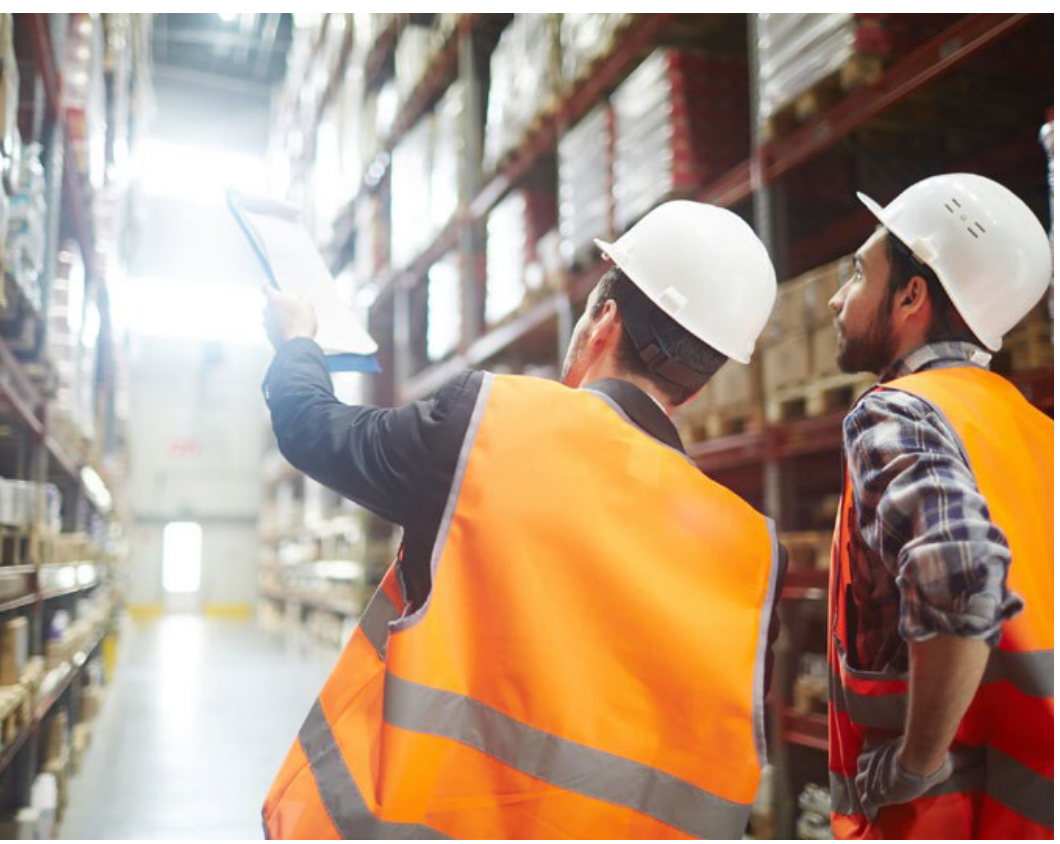

lugar que le corresponde, pudiendo así identificar riesgos de trabajo y de esta forma actuar en consecuencia.

Cabe aclarar que los resultados obtenidos mediante la investigación y que han sido analizados en este documento no pueden ser aplicables a todos los sectores ni ser generalizados para hacer inferencias sobre empresas que no tengan características similares a las que el presente estudio plantea; sino que buscan ser parte de una fuente de referencia que sirva de base para trabajos similares posteriores.

\section{BIBLIOGRAFÍA}

[1] Cochran, W. G. (1980). Técnicas de muestreo (p. 42). Editorial CECSA.

[2] Evaluación y prevención de riesgos. Biblioteca Técnica, Prevención de Riesgos Laborales. (2000). (p. 23). Ediciones CEAC.

[3] Grimaldi, J. V., Saldaña I. y Simonds, R. H. (2001). La seguridad industrial su administración. México D.F. (México: Alfaomega).

[4] Jelambi, O. (1967). Higiene y seguridad ocupacionales. Venezuela: Universidad Central de Venezuela.

[5] Montgomery, D. C. (1990). Control Estadístico de la Calidad (p. 442). Grupo Editorial Iberoamérica.

[6] Normas Oficiales Mexicanas. Disponible en: http://www. stps.gob.mx/02_sub_trabajo/01_dgaj/normas.htm

[7] Organización Internacional del Trabajo. (2019). Obtenido de Organización Internacional del Trabajo: https:// www.ilo.org/global/topics/safety-and-health-at-work/ lang--es/index.htm

[8] Reglamento Federal de Seguridad y Salud en el Trabajo. (2015). Disponible en: http://www.diputados.gob.mx/ LeyesBiblio/regla/n152.pdf

[9] Reglamento para la clasificación de empresas y determinación de la prima en el seguro de riesgos de trabajo. (1998). Editorial Offset, S. A. de C.V.

[10] Scheaffer, R., Mendenhall, W., y Ott, R. (2013). Elementos de muestreo. Madrid, España: Paraninfo.

[11] Secretaría de Economía México (2018). Disponible en: http://www.economia.gob.mx

[12] Secretaria del Trabajo y Previsión Social. (2017). Información sobre Accidentes y Enfermedades de Trabajo Sonora 2017. Disponible en: https:// autogestionsst.stps.gob.mx/Proyecto/Content/pdf/ Estadisticas/Sonora.pdf

[13] Secretaria del Trabajo y Previsión Social. (2017). Información sobre Accidentes y Enfermedades de Trabajo Nacional 2005-2017 Disponible en: https:// autogestionsst.stps.gob.mx/Proyecto/Content/pdf/ Estadisticas/Nacional.pdf

[14] Secretaría del Trabajo, Seguridad y Previsión Social. (2015). Normas Oficiales Mexicanas sobre Seguridad e Higiene Disponible en: http://www.stps.gob.mx/bp/ secciones/dgsst/normatividad/normas/Nom-001.pdf

[15] Secretaría del Trabajo, Seguridad y Previsión Social. (2015). Normas Oficiales Mexicanas sobre Seguridad e Higiene. Disponible en: http://www.stps.gob.mx/bp/ secciones/dgsst/normatividad/normas/Nom-002.pdf

[16] Secretaría del Trabajo, Seguridad y Previsión Social. (2015). Normas Oficiales Mexicanas sobre Seguridad e Higiene Disponible en: http://www.stps.gob.mx/bp/ secciones/dgsst/normatividad/normas/Nom-017.pdf

[17] Secretaría del Trabajo, Seguridad y Previsión Social. (2015). Normas Oficiales Mexicanas sobre Seguridad e Higiene. Disponible en: http://www.stps.gob.mx/bp/ secciones/dgsst/normatividad/normas/Nom-021.pdf 


\section{ANEXOS}

A1.

Clasificación de los riesgos laborales, según el Reglamento del IMSS

\begin{tabular}{|c|c|}
\hline CLASE & PRIMA MEDIA EN POR CIENTOS \\
\hline I & 0.54355 \\
\hline II & 1.13065 \\
\hline III & 2.5984 \\
\hline IV & 4.65325 \\
\hline V & 7.58875 \\
\hline
\end{tabular}

A2.

Formato de la encuesta

NOM-001-STPS-2008 Edificios, locales, instalaciones y áreas en los centros de trabajo Condiciones de seguridad.

\section{Recorrido por la empresa:}

1. ¿Cuenta su empresa con los registros de las verificaciones oculares realizadas al centro de trabajo, para identificar condiciones inseguras y reparar daños encontrados?

2. ¿Se encuentran en condiciones de uso, limpios y seguros, los sanitarios, retretes, mingitorios y lavabos?

3. ¿Se encuentren en condiciones de uso, limpios y seguros, los lugares reservados para el consumo de alimentos?

4. ¿Se encuentran físicamente que las escaleras, rampas, escaleras manuales, puentes y plataformas elevadas se encuentran en condiciones tales que en condiciones normales de uso los trabajadores no podrían resbalar?

5. ¿Se constata físicamente que los elementos estructurales que, del edificio, no presentan deformaciones o daños por las cargas que soportan, sin importar si han sido o no remodelados?

6. ¿Se observa que en los pisos de las diferentes áreas no se encuentren objetos, materiales o sustancias que sean un factor de riesgo para los trabajadores, de acuerdo a la naturaleza de las actividades que ahí se desarrollan?

7. ¿Se observa que las escaleras de emergencia exteriores cuentan en cada piso del edificio con un acceso al mismo nivel?

8. ¿Se constata que los sistemas de ventilación artificial son puestos en operación antes de que los trabajadores ingresen al área donde se encuentran las salidas de aire?

NOM-002-STPS-2010 Condiciones de seguridad Prevención y protección contra incendios en los centros de trabajo.

\section{Incendios:}

9. ¿Se observa que el ancho de las puertas de circulación de vehículos es superior al vehículo más grande que por ellas circule?

10. ¿Se ha elaborado un cálculo para la determinación final del riesgo de incendio?

11. ¿Se cuenta con un croquis, plano o mapa general del centro de trabajo, o por áreas que lo integran, tales como plantas, edificios o niveles, actualizado y colocado en los principales lugares de entrada, tránsito, reunión o puntos comunes de estancia o servicios para los trabajadores?

NOM-017-STPS-2008 Equipo de protección personal Selección, uso y manejo en los centros de trabajo.

\section{Equipo protección:}

12. ¿Se cuenta con un programa anual y/o mensual de revisión de los extintores?

13. ¿Cuenta con los registros de la identificación y análisis de los riesgos, por cada puesto de trabajo y área del centro laboral?

14. ¿Proporcionó a los trabajadores capacitación para que usen el equipo de protección personal de forma adecuada y con las limitaciones que el fabricante les establezca?

15. ¿Cuenta con registros de que proporciona equipo de protección personal a los trabajadores?

NOM-021-STPS-1993 Relativa a los requerimientos y características de los informes de los riesgos de trabajo que ocurran, para integrar las estadísticas.

\section{Estadísticas:}

16. ¿Cuenta con registros de que comunica a los trabajadores los riesgos de trabajo a los que están expuestos?

17. ¿Se cuenta con un registro de los avisos de los riesgos de trabajo ocurridos?

18. ¿Se hace del conocimiento de la Comisión Mixta de Seguridad e Higiene, los accidentes de trabajo que ocurren, o enfermedades que se detecten?

19. ¿Se llevan estadísticas de los accidentes de trabajo?

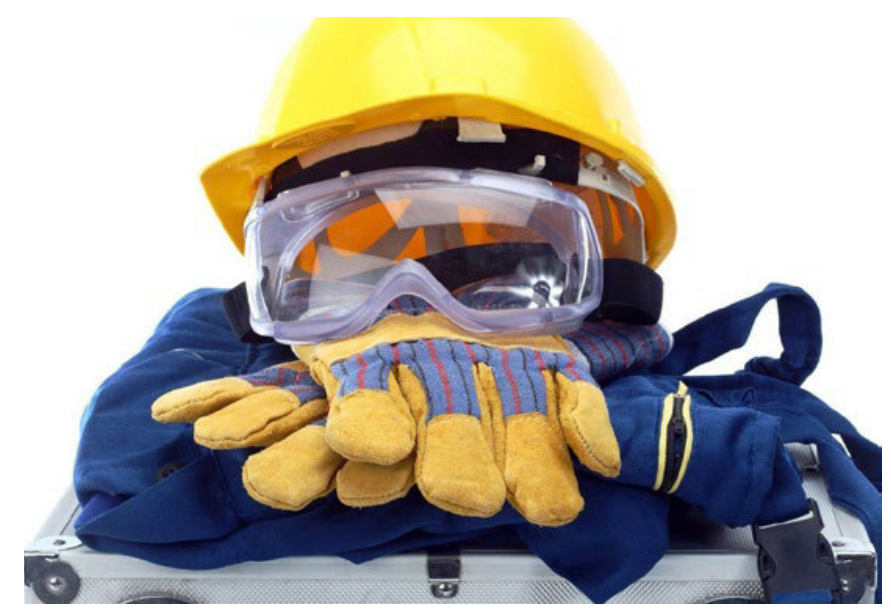

